\title{
Traffic phenomena in biology: from molecular motors to organisms*
}

\author{
Debashish Chowdhury \\ Department of Physics, Indian Institute of Technology, Kanpur 208016, India. \\ Andreas Schadschneider \\ Institut für Theoretische Physik, Universität zu Köln D-50937 Köln, Germany \\ Katsuhiro Nishinari \\ Department of Aeronautics and Astronautics, Faculty of Engineering, \\ University of Tokyo, Hongo, Bunkyo-ku, Tokyo 113-8656, Japan.
}

\begin{abstract}
Traffic-like collective movements are observed at almost all levels of biological systems. Molecular motor proteins like, for example, kinesin and dynein, which are the vehicles of almost all intracellular transport in eukayotic cells, sometimes encounter traffic jam that manifests as a disease of the organism. Similarly, traffic jam of collagenase MMP-1, which moves on the collagen fibrils of the extracellular matrix of vertebrates, has also been observed in recent experiments. Trafficlike movements of social insects like ants and termites on trails are, perhaps, more familiar in our everyday life. Experimental, theoretical and computational investigations in the last few years have led to a deeper understanding of the generic or common physical principles involved in these phenomena. In particular, some of the methods of non-equilibrium statistical mechanics, pioneered almost a hundred years ago by Einstein, Langevin and others, turned out to be powerful theoretical tools for quantitative analysis of models of these traffic-like collective phenomena as these systems are intrinsically far from equilibrium. In this review we critically examine the current status of our understanding, expose the limitations of the existing methods, mention open challenging questions and speculate on the possible future directions of research in this interdisciplinary area where physics meets not only chemistry and biology but also (nano-)technology.
\end{abstract}

\section{INTRODUCTION}

Motility is the hallmark of life. What distinguishes a traffic-like movement from all other forms of movements is that motile elements move on "tracks" or "trails". However, in sharp contrast to vehicular traffic, the tracks and trails, which are the biological analogs of roads, can have nontrivial dependence on time during the typical travel time of the motile elements. What makes biological traffic even more unusual is that in many cases the motile elements themselves not only create the tracks but also modify their lengths as well as shape and, in some extreme cases, even leave behind a trail of destruction by wiping out the track as they move forward.

We are mainly interested in the general principles and common trends seen in the mathematical modeling of collective traffic-like movements at different levels of biological organization [1]. Although the choice of the physical examples and modelling strategies are biased by our own works and experiences, we put these in a broader perspective by relating these with works of other research groups. We begin at the lowest level, starting with intracellular biomolecular motor traffic on filamentary rails. Then we present brief summaries of recent works on the traffic of molecular motors along the collagen fibrils in the extra-cellular matrix and those on transport of micron-size cargo by uni-cellular micro-organisms. We end our review by discussing the collective traffic-like terrestrial movements of social insects, particularly, ants, on their trails.

\section{THEORETICAL APPROACHES}

In recent years many individual-based models of biological traffic have been formulated in discretized space. While in some models the dynamics of the system has been formulated in terms of differential equations assuming continuous time, in many others the dynamics has been implemented in terms of "update rules" in discrete time steps in the spirit of cellular automata and lattice gas models [2, 3, , 4] . Most of these models are extensions of a class of particlehopping models which were earlier successfully used in the context of vehicular traffic [5]. The asymmetric simple exclusion process (ASEP) [6] is one of the simplest particle-hopping models. In the ASEP particles can hop (with some

\footnotetext{
* Based on a plenary lecture delivered by DC at TGF05, Berlin.
} 
probability or rate) from one lattice site to a neighbouring one, but only if the target site is not already occupied by another particle. "Simple Exclusion" thus refers to the absence of multiply occupied sites. Generically, it is assumed that the motion is "asymmetric" such that the particles have a preferred direction of motion.

For such driven diffusive systems the boundary conditions turn out to be crucial. If periodic boundary conditions are imposed, i.e., the sites 1 and $L$ are made nearest-neibours of each other, all the sites are treated on the same footing. If the boundaries are open, then a particle can enter from a reservoir and occupy the leftmost site $(j=1)$, with probability $\alpha$, if this site is empty. In this system a particle that occupies the rightmost site $(j=L)$ can exit with probability $\beta$. The ASEP has been studied extensively in recent years and is now well understood [6, 7]. Both parallel and random-sequential updating schemes have been studied extensively in the literature.

The average number of motile elements that arrive at (or depart from) a fixed detector site on the track per unit time interval is called the flux. One of the most important transport properties is the relation between the flux and the density of the motile elements; a graphical representation of this relation is usually referred to as the fundamental diagram. If the motile elements interact mutually only via their steric repulsion their average speed $v$ would decrease with increasing density because of the hindrance caused by each on the following elements. On the other hand, for a given density $c$, the flux $J$ is given by $J=c v(c)$, where $v(c)$ is the corresponding average speed. At sufficiently low density, the motile elements are well separated from each other and, consequently, $v(c)$ is practically independent of $c$. Therefore, $J$ is approximately proportional to $c$ if $c$ is very small. However, at higher densities the increase of $J$ with $c$ becomes slower. At high densities, the sharp decrease of $v$ with $c$ leads to a decrease, rather than increase, of $J$ with increasing $c$. Naturally, the fundamental diagram of such a system is expected to exhibit a maxium at an intermediate value of the density.

\section{INTRA-CELLULAR TRAFFIC OF CYTOSKELETAL MOLECULAR MOTORS}

Intracellular transport is carried by molecular motors which are proteins that can directly convert the chemical energy into mechanical energy required for their movement along filaments constituting what is known as the cytoskeleton [8, 9]. Three superfamilities of these motors are kinesin, dynein and myosin; majority of these motors are two-headed. Most of the kinesins and dyneins are like "porters" in the sense that these move over long distances carrying cargo along the filamentary tracks without getting completely detached; such motors are called processive. On the other hand, the conventional myosins and a few unconventional ones are nonprocessive; they are like "rowers".

These cytoskeleton-based molecular motors play crucially important biological functions, e.g., in axonal transport in neurons. The mechano-chemistry of single cytoskeletal motors and the mechanism of their motility have been investigated both experimentally and theoretically for quite some time [10, 11, 12].

Often a single microtubule (MT) is used simultaneously by many motors and, in such circumstances, the intermotor interactions cannot be ignored. In this article we shall focus mostly on the effects of mutual interactions of these motors on their collective spatio-temporal organisation and the biomedical implications of such organisations. Fundamental understanding of these collective physical phenomena may also expose the causes of motor-related diseases (e.g., Alzheimer's disease) 13, 14, 15, 16] thereby helping, possibly, also in their control and cure. The biomolecular motors have opened up a new frontier of applied research — "bio-nanotechnology". A clear understanding of the mechanisms of these natural nano-machines will give us clue as to the possible design principles that can be utilized to synthesize artificial nanomachines.

Derenyi and collaborators [17, 18] developed one-dimensional models of interacting Brownian motors. They modelled each motor as a rigid rod and formulated the dynamics through Langevin equations for each such rod assuming the validity of the overdamped limit; the mutual interactions of the rods were incorporated through the mutual exclusion.

The model considered by Aghababaie et al. 19] is not based on TASEP, but its dynamics is a combination of Brownian ratchet and update rules in discrete time steps. In this model the filamentary track is discretized and the motors are represented by field-driven particles in the spirit of the particle-hopping models. The hopping probabilities of the particles are obtained from the instantaneous form of the local time-dependent potential. No site can accomodate more than one particle at a time. Each time step consists of either an attempt of a particle to hop to a neighbouring site or an attempt that can result in switching of the potential from flat to sawtooth form or vice-versa. Both forward and backward movement of the particles are possible. However, neither attachment of new particles nor complete detachment of existing particles were allowed.

The fundamental diagram of the model [19], computed imposing periodic boundary conditions, is very similar to those of TASEP. This observation indicates that further simplification of the model proposed in ref.[19] is possible to develope a minimal model for interacting molecular motors. Indeed, the detailed Brownian ratchet mechanism, which leads to a noisy forward-directed movement of the field-driven particles in the model of Aghababaie et al. [19], is replaced in some of the more recent theoretical models [20, 21, 22, 23, 24, 25, 26, 27, 28] by a TASEP-like 
probabilistic forward hopping of self-driven particles [29]. In these simplied versions, none of the particles is allowed to hop backward and the forward hopping probability is assumed to capture most of the effects of biochemical cycle of the enzymatic activity of the motor. The explicit dynamics of the model is essentially an extension of that of the asymmetric simple exclusion processes (ASEP) [6, 30] that includes, in addition, Langmuir-like kinetics of adsorption and desorption of the motors.

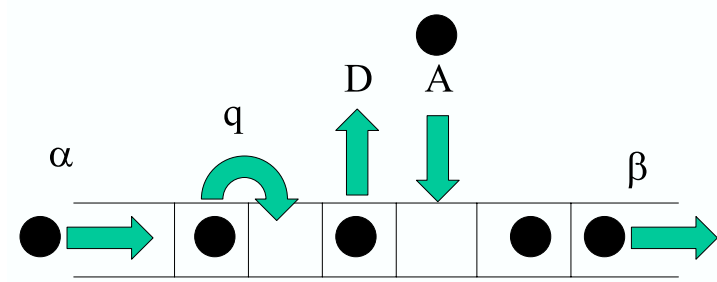

FIG. 1: A schematic description of the TASEP-like model introduced in ref. 24] for molecular motor traffic. Just as in TASEP, the motors are allowed to hop forward, with probability $q$. In addition, the motors can also get "attached" to an empty lattice site, with probability $A$, and "detached" from an occupied site, with probability $D$ from any site except the end points; the rate of attachment at the entry point on the left is $\alpha$ while that at the exit point on the right is $\beta$.

In the model of Parmeggiani et al. 24], the molecular motors are represented by particles whereas the sites for the binding of the motors with the cytoskeletal tracks (e.g., microtubules) are represented by a one-dimensional discrete lattice. Just as in TASEP, the motors are allowed to hop forward, with probability $q$, provided the site in front is empty. However, unlike TASEP, the particles can also get "attached" to an empty lattice site, with probability $A$, and "detached" from an occupied site, with probability $D$ (see fig:1) from any site except the end points. The state of the system was updated in a random-sequential manner. Carrying out Monte-Carlo simulations of the model, applying open boundary conditions, Parmeggiani et al.[24] demonstrated a novel phase where low and high density regimes, separated from each other by domain walls, coexist [26, 27]. Using a mean-field theory (MFT), they interpreted this spatial organization as traffic jam of molecular motors.

A cylindrical geometry of the model system was considered by Lipowsky, Klumpp and collaborators 20, 21, 22] to mimic the microtubule tracks in typical tubular neurons. The microtubule filament was assumed to form the axis of the cylinder whereas the free space surrounding the axis was assumed to consist of $N_{c h}$ channels each of which was discretized in the spirit of lattice gas models. They studied concentration profiles and the current of free motors as well as those bound to the filament by imposing a few different types of boundary conditions. This model enables one to incorporate the effects of exchange of populations between two groups, namely, motors bound to the axial filament and motors which move diffusively in the cylinder. They have also compared the results of these investigations with the corresponding results obtained in a different geometry where the filaments spread out radially from a central point.

A novel feature of the model of Klein et al. [31] (see Fig. 21) is that the lattice site at the tip of a filament is removed with a probability $W$ per unit time provided it is occupied by a motor; the motor remains attached to the newly exposed tip of the filament with probability $p$ (or remains bound with the removed site with probability $1-p$ ). Thus, $p$ may be taken as a measure of the processivity of the motors. This model clearly demonstrated a dynamic accumulation of the motors at the tip of the filament arising from the processivity.

\section{A. Traffic of interacting single-headed motors KIF1A}

The models of intracellular traffic described so far are essentially extensions of the asymmetric simple exclusion processes (ASEP) [6, 30] that includes Langmuir-like kinetics of adsorption and desorption of the motors. In reality, a motor protein is an enzyme whose mechanical movement is loosely coupled with its biochemical cycle. In a recent work [32], we have considered specifically the single-headed kinesin motor, KIF1A [33, 34, 35, 36, 37]; the movement of a single KIF1A motor was modelled earlier with a Brownian ratchet mechanism [38, 39]. In contrast to the earlier models 21, 24, 26, 28] of molecular motor traffic, which take into account only the mutual interactions of the motors, our model explicitly incorporates also the Brownian ratchet mechanism of individual KIF1A motors, including its biochemical cycle that involves adenosine triphosphate(ATP) hydrolysis.

The ASEP-like models successfully explain the occurrence of shocks. But since most of the bio-chemistry is captured in these models through a single effective hopping rate, it is difficult to make direct quantitative comparison with experimental data which depend on such chemical processes. In contrast, the model we proposed in ref. 32. 


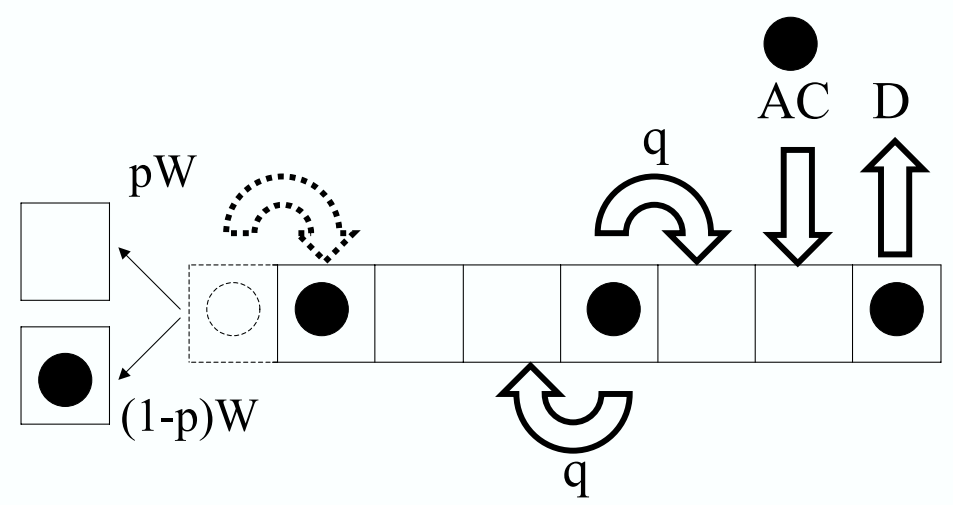

FIG. 2: A schematic description of the model suggested by Klein et al. [31] for motor induced depolymerization of cytoskeletal filaments.

incorporates the essential steps in the biochemical processes of KIF1A as well as their mutual interactions and involves parameters that have one-to-one correspondence with experimentally controllable quantities. Thus, in contrast to the earlier ASEP-like models, each of the self-driven particles, which represent the individual motors KIF1A, can be in two possible internal states labelled by the indices 1 and 2. In other words, each of the lattice sites can be in one of three possible allowed states (Fig. 3): empty (denoted by 0), occupied by a kinesin in state 1, or occupied by a kinesin in state 2 .

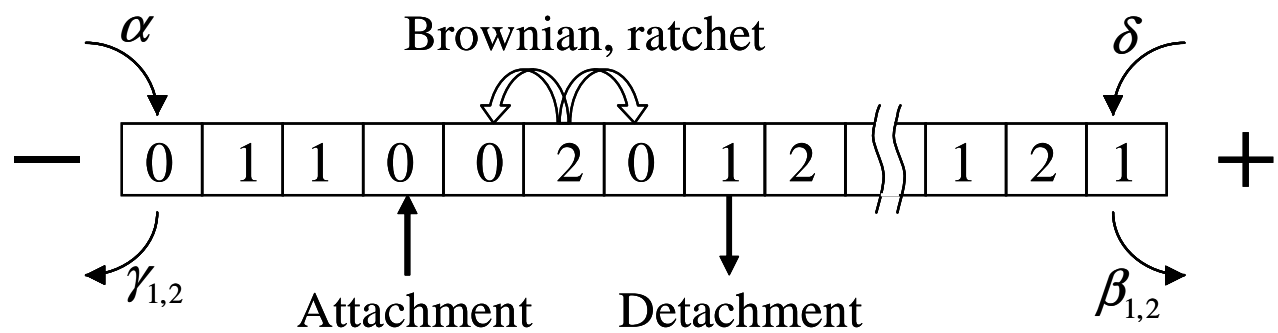

FIG. 3: A 3-state model for molecular motors moving along a MT. 0 denotes an empty site, 1 is $\mathrm{K}$ or KT and 2 is KD. Transition from 1 to 2, which corresponds to hydrolysis, occurs within a cell whereas movement to the forward or backward cell occurs only when motor is in state 2 . At the minus and plus ends the probabilities are different from those in the bulk.

Good estimates for the parameters of the model could be extracted by analyzing the empirical data [32]. Assuming that each time step of updating corresponds to $1 \mathrm{~ms}$ of real time, we performed simulations upto 1 minute. In the limit of low density of the motors we have computed, for example, the mean speed of the kinesins, the diffusion constant and mean duration of the movement of a kinesin on a microtubule from simulations of our model; these are in excellent quantitative agreement with the corresponding empirical data from single molecule experiments.

Using this model we have also calculated the flux of the motors in the mean field approximation imposing periodic boundary conditions. Although the system with periodic boundary conditions is fictitious, the results provide good estimates of the density and flux in the corresponding system with open boundary conditions.

In contrast to the phase diagrams in the $\alpha-\beta$-plane reported by earlier investigators [21, 25, 26], we have drawn the phase diagram of our model in the $\omega_{a}-\omega_{h}$ plane by carrying out extensive computer simulations for realistic parameter values of the model with open boundary conditions. The phase diagram shows the strong influence of hydrolysis on the spatial distribution of the motors along the MT. In particular, the position of the immobile shock depends on the concentration of the motors as well as that of ATP; the shock moves towards the minus end of the MT 
with the increase of the concentration of kinesin or ATP or both. The formation of the shock has been established by our direct experimental evidence; our findings on the domain wall are in qualitative agreement with the corresponding experimental observations [32].

This work has been discussed in more detailed in our separate article [40] in this proceedings.

\section{INTRA-CELLULAR TRAFFIC OF NUCLEOTIDE-BASED MOTORS}

Helicases and polymerases are the two classes of nucleotide-based motors that have been the main focus of experimental investigations. In this section, we discuss only the motion of the ribosome along the m-RNA track. Historically, this problem is one of the first where TASP-like model was successfully applied to a biological system.

In a living cell ribosomes translate the genetic information 'stored' in the messenger-RNA (mRNA) into a program for the synthesis of a protein. mRNA is a long (linear) molecule made up of a sequence of triplets of nucleotides; each triplet is called a codon (see Fig. (4). The genetic information is encoded in the sequence of codons. A ribosome, that first gets attached to the mRNA chain, "reads" the codons as it translocates along the mRNA chain, recruits the corresponding amino acids and assembles these amino acids in the sequence thereby synthesizing the protein for which the "construction plan" was stored in the mRNA. Once the synthesis is completed, the ribosome gets detached from the mRNA. Thus, the process of "translation" of genetic information stored in mRNA consists of three steps: (i) initiation: attachment of a ribosome at the "start" end of the mRNA, (ii) elongation: of the polypeptide (protein) as the ribosome moves along the mRNA, and (iii) termination: ribosome gets detached from the mRNA when it reaches the "stop" codon.

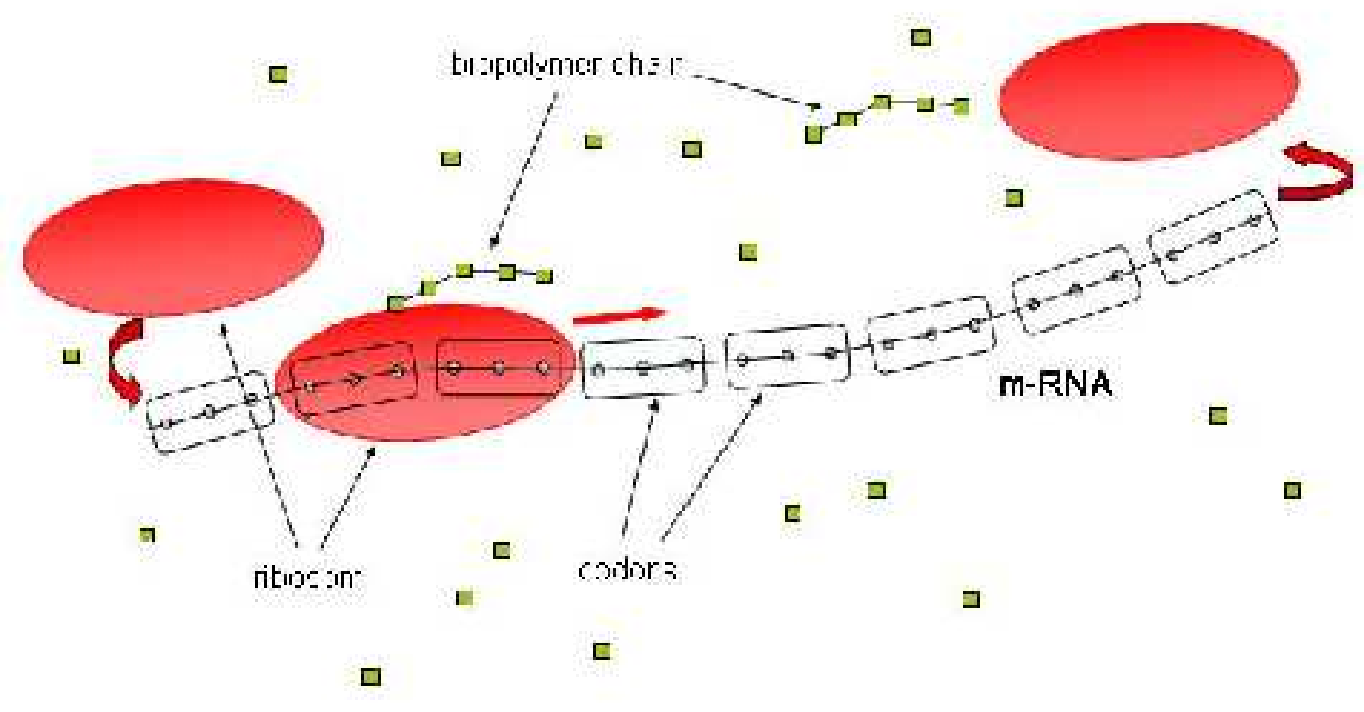

FIG. 4: The process of biopolymerization: Ribosomes attach to mRNA and read the construction plan for a biopolymer which is stored in the genetic code formed by the sequence of codons. None of the codons can be read by more than one ribosome simultaneously.

In order to model the traffic of ribosomes on a m-RNA track, let us denote each of the successive codons by the successive sites of a one-dimensional lattice where the first and the last sites correspond to the start and stop codons. The ribosomes are much bigger (20-30 times) than the codons. Therefore, neighbouring ribosomes attached to the same mRNA can not read the same information or overtake each other. In other words, any given site on the lattice may be covered by a single ribosome or none. Let us represent each ribosome by a rigid rod of length $\ell_{r}$. If the rod 


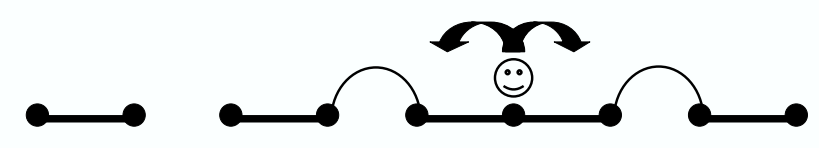

FIG. 5: Schematic representation of the one-dimensional burnt bridge model of MMP-1 dynamics proposed in ref. 50.

representing the ribosome has its left edge attached to the i-th site of the lattice, it is allowed to move to the right by one lattice spacing, i.e., its left edge moves to the site $i+1$ provided the site $i+\ell_{r}$ is empty. In the special case $\ell_{r}=1$ this model reduced to the TASEP. Although the model was originally proposed in the late sixties [41], significant progress in its analytical treatment for the general case of arbitrary $\ell_{r}$ could be made only three decades later; even the effects of quenched disorder has also been considered in the recent literature [42, 43, 44, 45, 46].

As mentioned above, a ribosom is much bigger than a base triplet. However, modifying the ASEP by taking into account particles that occupy more than one lattice site does not change the structure of phase diagram [41].

\section{EXTRACELLULAR TRANSPORT: COLLAGEN-BASED MOTORS}

The extracellular matrix (ECM) [47] of vertebrates is rich in collagen. Monomers of collagen form a triple-helical structure which self-assemble into a tightly packed periodic organization of fibrils. Cells residing in tissues can secret matrix metalloproteases (MMPs), a special type of enzymes that are capable of degrading macromolecular constituents of the ECM. The most notable among these enzymes is MMP-1 that is known to degrade collagen. The collagen fibril contains cleavage sites which are spaced at regular intervals of $300 \mathrm{~nm}$. The collagenase MMP-1 cleaves all the three $\alpha$ chains of the collagen monomer at a single site.

Breakdown of the ECM forms an essential step in several biological processes like, for example, embryonic development, tissue remodelling, etc. [47]. Malfunctioning of MMP-1 has been associated with wide range of diseases [48]. Therefore, an understanding of the MMP-1 traffic on collagen fibrils can provide deeper insight into the mechanism of its operation which, in turn, may give some clue as to the strategies of control and cure of diseases caused by the inappropriate functions of these enzymes.

Saffarian et al. [49] used a technique of two-photon excitation fluorescence correlation spectroscopy to measure the correlation function corresponding to the MMP-1 moving along the collagen fibrils. The measured correlation function strongly indicated that the motion of the MMP-1 was not purely diffusive, but a combination of diffusion and drift. In other words, the "digestion" of a collagen fibril occurs when a MMP-1 executes a biased diffusion processively (i.e., without detachment) along the fibril. They also demonstrated that inactivation of the enzyme eliminates the bias but the diffusion remains practically unaffected. They claimed that the energy required for the active motor-like transport of the MMP-1 comes from the proteolysis (i.e., degradation) of the collagen fibrils.

There is a close relation between the traffic of MMP-1 on collagens and the "burnt-bridge model" introduced by Mai et al. [50]. In the burnt bridge model (see Fig. 5), a "particle" performs a random walk on a semi-infinite one-dimensional lattice that extends from the origin to $+\infty$. Each site of the lattice is connected to the two nearest neighbour sites by links; a fraction $c$ of these links are called "bridges" and these are prone to be burnt by the random walker. A bridge is burnt, with probability $p$, if the random walker either crosses it from left to right or attempts to cross if from right to left [50, 51]. In either case, if the bridge is actually burnt, the walker stays on the right of the burnt bridge and cannot cross it any more. The hindrance against leftward motion, that is created by the burnt bridges, is responsible for the overall rightward drift of the random walker. Mai et al. [50] studied the dependence of the average drift velocity $v$ on the parameters $p$ and $c$ by computer simulation. They also derived approximate analytical forms of these dependences in the two limits $p \ll 1$ and $p \simeq 1$ using a continuum approximation.

Saffarian et al. 49] also carried out computer simulations of a two-dimensional model of the MMP-1 dynamics on collagen fibrils which is essentially a two-dimensional generalization of the burnt-bridge model. By comparing the results of their simulations with their experimental observations, Saffarian et al. they concluded that the observed biased diffusion of the MMP-1 on collagen fibrils can be described quite well by a Brownian ratchet mechanism [38, 39]. 


\section{CELLULAR TRAFFIC}

A Mycoplasma mobile (MB) bacterium is an uni-cellular organism. Each of the pear-shaped cells of this bacterium is about $700 \mathrm{~nm}$ long and has a diameter of about $250 \mathrm{~nm}$ at the widest section. Each bacterium can move fast on glass or plastic surfaces using a gliding mechanism. In a recent experiment [52] narrow linear channels were constructed on lithographic substrates. The channels were typically $500 \mathrm{~nm}$ wide and $800 \mathrm{~nm}$ deep. Note that each channel was approximately twice as wide as the width of a single MB cell. The channels were so deep that none of the individual MB cells was able to climb up the tall walls of the channels and continued moving along the bottom edge of the walls of the cannels. In the absence of direct contact interaction with other bacteria, each individual MB cell was observed to glide, without changing direction, at an average speed of a few microns per second.

When two MB cells made a contact approaching each other from opposite directions within the same channel, one of the two cells gave way and moved to the adjacent lane. However, in a majority of the cases, two cells approaching each other from the opposite directions simply passed by as if nothing had happened; this is because of the fact that the width of the channel is roughly twice that of the individual MB cell. Moreover, when two cells moving in the same direction within a channel collided with each other, the faster cell moved to the adjacent lane after the collision.

Hiratsuka et al. 52] attached micron-sized beads on the MB cells using biochemical technique and demonstrated that the average speed of each MB cell remained practically unaffected by the load it was carrying. In contrast to the nonliving motile elements discussed in all the preceedings sections, the cells are the functional units of life. Therefore, the MB cells have the potential for use in applied research and technology as "micro-transporters". More recently, the unicellular biflagellated algae Chlamydomonas reinhardtii (CR), which are known to be phototactic swimmers, have been shown to be even better candidates as "micro-transporter" as these can attain average speeds that is about two orders of magnitude higher than what was possible with MB cells [53]. However, to our knowledge, the effects of mutual interactions of the CR cells on their average speed at higher densities has not been investigated.

\section{TRAFFIC IN SOCIAL INSECT COLONIES: ANTS AND TERMITES}

From now onwards, we shall study traffic of multi-cellular organisms, particularly, ants which are social insects. The ability of the social insect colonies to function without a leader has attracted the attention of experts from various disciplines $54,55,56,57,58,59,60,61$. Insights gained from the modeling of the colonies of such insects are finding important applications in computer science (useful optimization and control algorithms) [62], communication engineering [63], artificial "swarm intelligence" 64] and micro-robotics [65] as well as in task partitioning, decentralized manufacturing [66, 67, 68, 69, 70, 71] and management [72]. the collective terrestrial movements of ants have close similarities with the other traffic-like phenomena considered here. When observed from a sufficiently long distance the movement of ants on trails resemble the vehicular traffic observed from a low flying aircraft.

Ants communicate with each other by dropping a chemical (generically called pheromone) on the substrate as they move forward [73, 74, 75]. Although we cannot smell it, the trail pheromone sticks to the substrate long enough for the other following sniffing ants to pick up its smell and follow the trail. 73]. Both the continuum model developed by Rauch et al. 76] and the CA model introduced by Watmough and Edelstein-Keshet [77] were intended to address the question of formation of the ant-trail networks by foraging ants. Couzin and Franks [78] developed an individual based model that not only addressed the question of self-organized lane formation but also elucidated the variation of the flux of the ants.

In the recent years, we have developed discrete models $79,80,81,82$ that are not intended to address the question of the emergence of the ant-trail [83], but focus on the traffic of ants on a trail which has already been formed. We have developed models of both unidirectional and bidirectional ant-traffic by generalizing the totally asymmetric simple exclusion process (TASEP) [6, 84, 85] with parallel dynamics by taking into account the effect of the pheromone.

In our model of uni-directional ant-traffic the ants move according to a rule which is essentially an extension of the TASEP dynamics. In addition, a second field is introduced which models the presence or absence of pheromones (see Fig. 6). The hopping probability of the ants is now modified by the presence of pheromones. It is larger if a pheromone is present at the destination site. Furthermore, the dynamics of the pheromones has to be specified. They are created by ants and free pheromones evaporate with probability $f$ per unit time. Assuming periodic boundary conditions, the state of the system is updated at each time step in two stages. In stage I ants are allowed to move while in stage II the pheromones are allowed to evaporate. In each stage the stochastic dynamical rules are applied in parallel to all ants and pheromones, respectively.

One interesting phenomenon observed in the simulations is coarsening. At intermediate time usually several noncompact clusters are formed. However, the velocity of a cluster depends on the distance to the next cluster ahead. Obviously, the probability that the pheromone created by the last ant of the previous cluster survives decreases with 


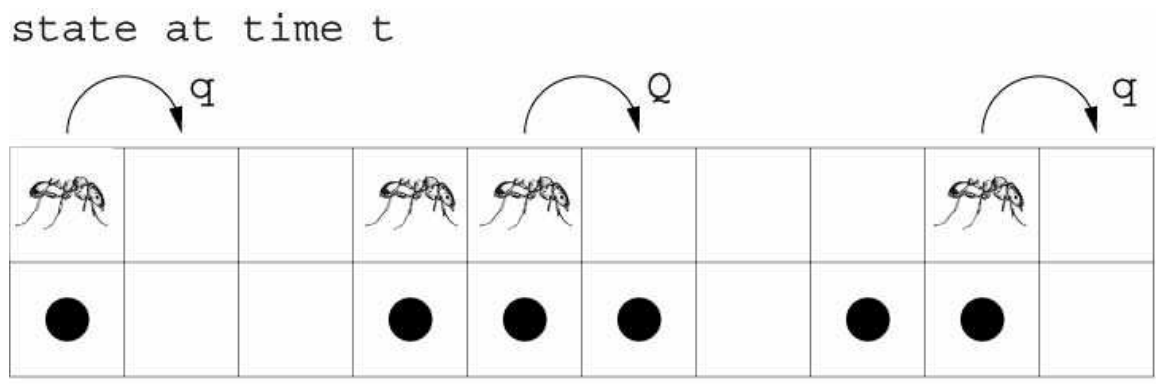

stage I

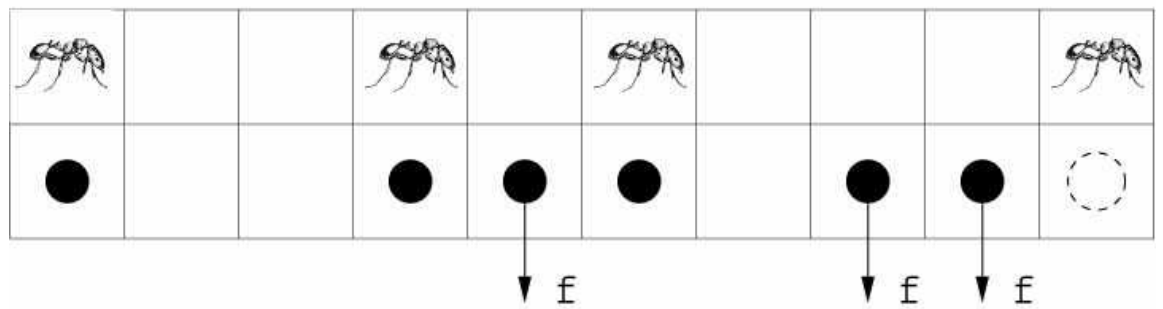

stage II

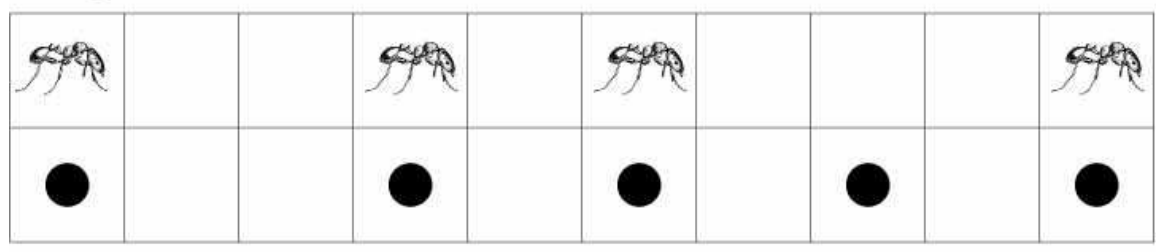

state at time $t+1$

FIG. 6: Schematic representation of typical configurations of the uni-directional ant-traffic model. The symbols $\bullet$ indicate the presence of pheromone. This figure also illustrates the update procedure. Top: Configuration at time $t$, i.e. before stage $I$ of the update. The non-vanishing probabilities of forward movement of the ants are also shown explicitly. Middle: Configuration after one possible realisation of stage I. Two ants have moved compared to the top part of the figure. The open circle with dashed boundary indicates the location where pheromone will be dropped by the corresponding ant at stage $I I$ of the update scheme. Also indicated are the existing pheromones that may evaporate in stage II of the updating, together with the average rate of evaporation. Bottom: Configuration after one possible realization of stage II. Two drops of pheromones have evaporated and pheromones have been dropped/reinforced at the current locations of the ants.

increasing distance. Therefore clusters with a small headway move faster than those with a large headway. This induces a coarsening process such that after long times only one non-compact cluster survives.

A similar coarsening phenomenon has been observed also in the bus-route model [86, 87]. In fact, the close relation between our model of uni-directional traffic on ant-trails and the bus-route model has been pointed out earlier [88]. In the bus route model, each bus stop can accomodate at most one bus at a time; the passengers arrive at the bus stops randomly at an average rate $\lambda$ and each bus, which normally moves from one stop to the next at an average rate $Q$, slows down to $q$, to pick up waiting passengers [86, 87].

In vehicular traffic, usually, the average speed of the vehicles decreases monotonically with increasing density because the inter-vehicle interactions tend to hinder each other's movements. In contrast, in our models of ant-traffic the average speed of the ants varies non-monotonically with their density over a wide range of small values of $f$ because of the coupling of their dynamics with that of the pheromone. This uncommon variation of the average speed gives rise to the unusual dependence of the flux on the density of the ants in our models of ant-traffic. Furthermore, the flux does not exhibit the particle-hole symmetry which is a characteristic of the TAEP. Details of the models and results on both uni-directional and bi-directional traffic of ants on trails are given in the article of John et al. in this proceedings [89]. The experimental data reported in the pioneering experimental work of Burd and collaborators [90] were too scattered to test our theoretical predictions. However, more accurate recent data [91, 92] establish both the 
non-monotonic variation of the average speed with density as well as the formation of cluster by the ants.

\section{SUMMARY AND CONCLUSION}

In this article we have reviewed our current understanding of traffic-like collective phenomena in living systems, starting from the smallest level of intra-cellular bio-molecular motor transport and ending at the level of the traffic of social insects like, for example, ants. We have restricted our attention to those theoretical works where, in the spirit of particle-hopping models of vehicular traffic, the language of cellular automata or extensions of TASEP has been used. The success of this modelling strategy has opened up a new horizon in traffic science and, we hope, we have provided a glimpse of an exciting frontier of interdisciplinary research.

Acknowledgements: It is our great pleasure to thank Yasushi Okada, Alexander John and Ambarish Kunwar for enjoyable collaborations on the topics discussed in this article.

[1] D. Chowdhury, A. Schadschneider and K. Nishinari, Phys. of Life Rev. (2005) in press.

[2] S. Wolfram, Theory and Applications of Cellular Automata (World Sci., 1986); A New Kind of Science (Wolfram Research Inc., 2002)

[3] B. Chopard and M. Droz, Cellular Automata Modeling of Physical Systems (Cambridge University Press, 1998).

[4] J. Marro and R. Dickman, Nonequilibrium Phase Transitions in Lattice Models (Cambridge University Press, 1999).

[5] D. Chowdhury, L. Santen, and A. Schadschneider, Phys. Rep. 329, 199 (2000).

[6] G.M. Schütz: Exactly Solvable Models for Many-Body Systems, in C. Domb and J.L. Lebowitz (eds.), Phase Transitions and Critical Phenomena, Vol. 19 (Academic Press, 2001).

[7] M.R. Evans and R.A. Blythe, Physica A313, 110 (2002).

[8] J. Howard, Mechanics of Motor Proteins and the Cytoskeleton, (Sinauer Associates, 2001) .

[9] M. Schliwa (ed.), Molecular Motors, (Wiley-VCH, 2002).

[10] G. Oster and H. Wang, in ref. [9].

[11] M.E. Fisher and A.B. Kolomeisky, Proc. Natl. Acad. Sci. 98, 7748 (2001).

[12] R.D. Astumian, Appl. Phys. A 75, 193 (2002).

[13] M. Aridor and L.A. Hannan, Traffic 1, 836 (2000); 3, 781 (2002).

[14] N. Hirokawa and R. Takemura, Trends in Biochem. Sci. 28, 558 (2003)

[15] E. Mandelkow and E.M. Mandelkow, Trends in Cell Biol. 12, 585 (2002).

[16] L.S. Goldstein, Proc. Natl. Acad. Sci. 98, 6999 (2001); Neuron 40, 415-425 (2003). 28, 558 (2003); Curr. Op. Neurobiol. 14, 564-573 (2004).

[17] I. Derenyi and T. Vicsek, Phys. Rev. Lett. 75, 374 (1995).

[18] I. Derenyi and A. Ajdari, Phys. Rev. E 54, R5 (1996).

[19] Y. Aghababaie, G.I. Menon and M. Plischke, Phys. Rev. E 59, 2578 (1999).

[20] R. Lipowksy, S. Klumpp, and Th. M. Nieuwenhuizen, Phys. Rev. Lett. 87, 108101 (2001).

[21] R. Lipowksy and S. Klumpp, Physica A 352, 53 (2005).

[22] M.J.I. Müller, S, Klumpp and R. Lipowsky, J. Phys. Cond. Matt. 17, S3839 (2005) and references therein.

[23] S. Klumpp and R. Lipowsky, this proceedings.

[24] A. Parmeggiani, T. Franosch, and E. Frey, Phys. Rev. Lett. 90, 086601 (2003); Phys. Rev. E 70, 046101 (2004).

[25] E. Frey, A. Parmeggiani and T. Franosch, Genome Informatics 15(1), 46 (2004) and references therein.

[26] M.R. Evans, R. Juhasz, and L. Santen, Phys. Rev. E 68, 026117 (2003).

[27] R. Juhasz and L. Santen, J. Phys. A 37, 3933 (2004).

[28] V. Popkov, A. Rakos, R.D. Williams, A.B. Kolomeisky, and G.M. Schütz, Phys. Rev. E 67, 066117 (2003).

[29] F. Schweitzer: Brownian Agents and Active Particles, Springer Series in Synergetics (Springer 2003).

[30] B. Schmittmann and R.P.K. Zia, in C. Domb and J.L. Lebowitz (eds.), Phase Transitions and Critical Phenomena, Vol. 17 (Academic Press, 1995).

[31] G.A. Klein, K. Kruse, G. Cuniberti and F. Jülicher, Phys Rev. Lett. 94, 108102 (2005).

[32] K. Nishinari, Y. Okada, A. Schadschneider and D. Chowdhury, Phys. Rev. Lett. 95, 118101 (2005).

[33] Y. Okada and N. Hirokawa, Science 283, 1152 (1999).

[34] Y. Okada and N. Hirokawa, Proc. Natl. Acad.Sci. USA 97, 640 (2000).

[35] Y. Okada, H. Higuchi, and N. Hirokawa, Nature, 424, 574 (2003).

[36] R. Nitta, M. Kikkawa, Y. Okada, and N. Hirokawa, Science 305, 678 (2003).

[37] Y. Okada, K. Nishinari, D. Chowdhury, A. Schadschneider, and N. Hirokawa (to be published).

[38] F. Jülicher, A. Ajdari, and J. Prost, Rev. Mod. Phys. 69, 1269 (1997). 
[39] P. Reimann, Phys. Rep. 361, 57-265 (2002).

[40] K. Nishinari, Y. Okada, A. Schadschneider and D. Chowdhury, in this proceedings.

[41] C. MacDonald, J. Gibbs, and A. Pipkin, Biopolymers 6, 1 (1968); C. MacDonald and J. Gibbs, Biopolymers 7, 707 (1969)

[42] L.B. Shaw, R.K.P. Zia and K.H. Lee, Phys. Rev. E 68, 021910 (2003).

[43] L.B. Shaw, J. P. Sethna and K.H. Lee, Phys. Rev. E 70, 021901 (2004).

[44] L.B. Shaw, A.B. Kolomeisky and K.H. Lee, J. Phys. A 37, 2105 (2004).

[45] G. Lakatos and T. Chou, J. Phys. A 36, 2027 (2003).

[46] T. Chou and G. Lakatos, Phys. Rev. Lett. 93, 198101 (2004).

[47] H. Nagase and J. F. Woessner, J. Biol. Chem. 274, 21491 (1999).

[48] M. Whittaker and A. Ayscough, Celltransmisions 17, 3 (2001).

[49] S. Saffarian, I. E. Collier, B.L. Marmer, E.L. Elson and G. Goldberg, Science 306, 108 (2004).

[50] J. Mai, I.M. Sokolov and A. Blumen, Phys. Rev. E 64, 011102 (2001).

[51] T. Antal and P.L. Krapivsky, cond-mat/0504652.

[52] Y. Hiratsuka, M. Miyata and T. Q. P. Uyeda, Biochem. Biophys. Res. Commun. 331, 318 (2005).

[53] D. B. Weibel, P. Garstecki, D. Ryan, W. R. DiLuzio, M. Mayer, J. E. Seto and G. M. Whitesides, Proc. Nat. Acad. Sci. USA, 102, 11963 (2005).

[54] E. Bonabeau, G. Theraulaz, J.L. Deneubourg, S. Aron and S. Camazine, Trends in Ecol. Evol. 12, 188 (1997)

[55] C. Anderson, G. Theraulaz and J.L. Deneubourg, Insect. Sociaux 49, 99 (2002)

[56] Z. Huang and J.H. Fewell, Trends in Ecol. Evol. 17, 403 (2002).

[57] E. Bonabeau, Ecosystems 1, 437 (1998).

[58] G. Theraulaz, J. Gautrais, S. Camazine and J.L. Deneubourg, Phil. Trans. Roy. Soc. Lond. A 361, 1263 (2003).

[59] J. Gautrais, G. Theraulaz, J.L. Deneubourg and C. Anderson, J. Theor. Biol. 215, 363 (2002).

[60] L. Edelstein-Keshet, J. Math. Biol. 32, 303 (1994).

[61] G. Theraulaz, E. Bonabeau, S.C. Nicolis, R.V. Sole, V. Fourcassie, S. Blanco, R. Fournier, J.L. Joly, P. Fernandez, A. Grimal, P. Dalle and J.L. Deneubourg, Proc. Natl.Acad. Sci. 99, 9645 (2002).

[62] M. Dorigo, G. di Caro and L.M. Gambardella, Artificial Life 5(3), 137 (1999); Special issue of Future Generation Computer Systems dedicated to ant-algorithms (2000).

[63] E. Bonabeau, M. Dorigo and G. Theraulaz, Nature 400, 39 (2000).

[64] E. Bonabeau, M. Dorigo and G. Theraulaz, Swarm Intelligence: From Natural to Artificial Intelligence (Oxford University Press, 1999).

[65] M.J.B. Krieger, J.B. Billeter and L. Keller, Nature 406, 992 (2000).

[66] F.L.W. Ratnieks and C. Anderson, Insectes Sociaux 46, 95 (1999).

[67] C. Anderson and F.L.W. Ratnieks, Am. Nat. 154, 521 (1999).

[68] F.L.W. Ratnieks and C. Anderson, Am. Nat. 154, 536 (1999).

[69] C. Anderson and F.L.W. Ratnieks, Insectes Sociaux 47, 198 (2000).

[70] C. Anderson and D.W. McShea, Biol. Rev. 76, 211 (2001).

[71] C. Anderson and F.L.W. Ratnieks, in: Complexity and complex systems in industry, eds. I.P. McCarthy and T. RakotobeJoel, (University of Warwick, U.K.), 92 (2000).

[72] E. Bonabeau and C. Meyer, Harvard Business Review (May), 107 (2001).

[73] E.O. Wilson, The Insect Societies (Belknap, Cambridge, USA, 1971); B. Hölldobler and E.O. Wilson, The Ants (Belknap, Cambridge, USA, 1990)

[74] S. Camazine, J.L. Deneubourg, N. R. Franks, J. Sneyd, G. Theraulaz, E. Bonabeau: Self-organization in Biological Systems (Princeton University Press, 2001).

[75] A.S. Mikhailov and V. Calenbuhr, From Cells to Societies: Models of Complex Coherent Action (Springer, 2002).

[76] E.M. Rauch, M. M. Millonas and D.R. Chialvo, Phys. Lett. A 207, 185 (1995).

[77] J. Watmough and L. Edelstein-Keshet, J. Theor. Biol. 176, 357 (1995).

[78] I.D. Couzin and N.R. Franks, Proc. Roy Soc. London B 270, 139 (2003).

[79] D. Chowdhury, V. Guttal, K. Nishinari, A. Schadschneider, J. Phys. A:Math. Gen. 35, L573 (2002)

[80] K. Nishinari, D. Chowdhury, A. Schadschneider, Phys. Rev. E 67, 036120 (2003)

[81] A. Kunwar, D. Chowdhury, A. Schadschneider and K. Nishinari, submitted for publication.

[82] A. John, A. Schadschneider, D. Chowdhury and K. Nishinari, J. Theor. Biol. 231, 279 (2004).

[83] D. Helbing, F. Schweitzer, J. Keltsch, P. Molnar: Phys. Rev. E56, 2527 (1997)

[84] B. Derrida, Phys. Rep. 301, 65 (1998)

[85] B. Derrida and M.R. Evans, in: Nonequilibrium Statistical Mechanics in One Dimension, ed. V. Privman (Cambridge University Press, 1997)

[86] O.J. O'Loan, M.R. Evans, M.E. Cates, Europhys. Lett. 42, 137 (1998); Phys. Rev. E58, 1404 (1998).

[87] D. Chowdhury, R.C. Desai, Eur. Phys. J. B15, 375 (2000).

[88] A. Kunwar, A. John, K. Nishinari, A. Schadschneider and D. Chowdhury, J. Phys. Soc. Jap. 73, 2979 (2004).

[89] A. John, A. Kunwar, A. Namazi, A. Schadschneider, D. Chowdhury, and K. Nishinari, in this proceedings.

[90] M. Burd, D. Archer, N. Aranwela and D.J. Stradling, Am. Nat. 159, 283 (2002).

[91] M. Burd et al. (2005) unpublished.

[92] A. John et al. (2005) unpublished. 


\title{
Focusing of a Parallel Beam to Form a Point in the Particle Deflection Plane
}

\author{
Ivar Ekeland ${ }^{1}$, Roger Temam ${ }^{2}$, Jeffrey Dean ${ }^{2}$, David Grove ${ }^{1}$, Craig Chambers ${ }^{2}$, \\ Kim B. Bruce ${ }^{2}$, and Elsa Bertino ${ }^{1}$ \\ 1 Princeton University, Princeton NJ 08544, USA \\ 2 Université de Paris-Sud, Laboratoire d'Analyse Numérique, Bâtiment 425, \\ F-91405 Orsay Cedex, France
}

\begin{abstract}
The abstract is optional. If present it should summarize the contents of the paper in at least 70 and at most 150 words; neither too long nor too short but to the point!
\end{abstract}

\section{Text}

Please consult the file or printout of 1 readme.* to find detailed instructions on the layout and style of text elements.

\subsection{Fixed-Period Problems: The Sublinear Case}

Of course this text has no scientific relevance but should be used as a practical example of the Springer layout specifications for physics books [3].

We begin now the search for periodic solutions to Hamiltonian systems. All this will be done in the convex case; that is, we shall study the boundary-value problem

$$
\begin{aligned}
& \dot{x}(0)=J H^{\prime}(t, x) \\
& x(0)=x(T)
\end{aligned}
$$

with $H(t, \cdot)$ a convex function of $x$, going to $+\infty$ when $\|x\| \rightarrow \infty$.

\section{Equations}

Please consult the file or printout of 1 readme.* to find detailed instructions on the layout and style of mathematical elements.

The following equation is the compiled result of using the command Ivec and using the newly defined command \umu:

$$
\boldsymbol{A}=\mu y=50 \mu \mathrm{m} .
$$




\section{$2.1 \quad$ Sub-equations}

As you have seen above, equations are numbered automatically when the equations are defined with \begin\{equation\} (but not when } \$ \text { \$ is used). Here you } will find an example for the automatic sub-numbering of equation arrays, using the style subeqnar. sty:

$$
\begin{aligned}
& a=c+d, \\
& e=f-d .
\end{aligned}
$$

In order to refer to the equations within the main text you must simply label your equations (within the equation's environment) and quote the labels within the text environment. Upon running $\mathrm{T}_{\mathrm{E}} \mathrm{X}$ you should receive the corresponding subnumbers, e.g. (4a) and (4b).

No Sub-numbering. If you want to suppress the sub-numbering of an equation array you may use the original \begin\{subeqnarray\} environment, put } \nonumber at the end of the line that is to have no number, and set the subcounter to zero:

$$
\begin{aligned}
& a=c+d, \\
& e=f-d .
\end{aligned}
$$

\section{$3 \quad$ Figures}

Please consult the file or printout of 1 readme.* to find detailed instructions on how to treat figures.

If your figures are available as electronic data it is advisable to convert them to eps-format and include them directly into the text with the help of the graphicx package (see the following example in Fig. 1)

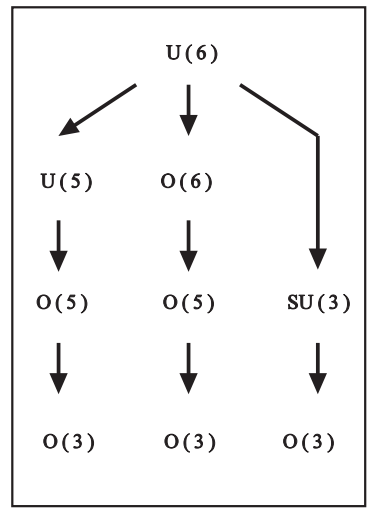

Fig. 1. Example of an electronically included eps-figure 
In order to mark the desired amount of space for a (centered!) figure which has to be pasted into the manuscript manually please provide a vertical line on the lefthand side of the figure. This is reached by using the commands $\backslash$ mpicplace width in $\mathrm{cm}\}$ height in $\mathrm{cm}$ \}.

For further instructions e.g. on the structure and layout of the caption see the captions of Figs. 2 and 3.

$$
\mid
$$

Fig. 2. General description. (a) The 'name' of the sub-figure should be set boldface and in round brackets. (b) In general the last sentence of a figure caption should not end with a fullstop

Fig. 3. This could show a figure consisting of different types of lines to describe individual aspects. These descriptive lines (dotted line) should be set italic and put into round brackets as shown in this sample figure caption. In general the last sentence of a figure caption (straight line) should not end with a fullstop

\section{Tables}

If equations and figures are centered then tables should be centered as well. Table captions should be treated in the same way as figure legends, except that the table captions appear above the tables. Overwide tables should be reduced to the page width, if possible, or exceed the type area by a maximum of $5 \mathrm{~mm}$. Please check the $\mathrm{T}_{\mathrm{E}} \mathrm{X}$ files of the following tables (Table 1 and 2) and use them as an example for setting your own tables. 
Table 1. Critical $N$ values

\begin{tabular}{llllll}
\hline $\mathrm{M}_{\odot}$ & $\beta_{0}$ & $T_{\text {c6 }}$ & $\gamma$ & $N_{\text {crit }}^{\mathrm{L}}$ & $N_{\text {crit }}^{\mathrm{Te}}$ \\
\hline 30 & 0.82 & 38.4 & 35.7 & 154 & 320 \\
60 & 0.67 & 42.1 & 34.7 & 138 & 340 \\
120 & 0.52 & 45.1 & 34.0 & 124 & 370 \\
\hline
\end{tabular}

Table 2. Please write your table caption here. Multi-line captions as well as single-line captions automatically will be set flushleft

\begin{tabular}{llll}
\hline $\begin{array}{l}\text { Nominal dimension } \\
(\mathrm{mm})\end{array}$ & Angle & $\begin{array}{l}\text { Tolerance } \\
(\mu \mathrm{m})\end{array}$ & Evaluation factor \\
\hline $10^{1}$ & $1^{\circ}$ & $\begin{array}{l}\text { Untoleranced } \\
\text { dimension }\end{array}$ & $c_{1}=0$ \\
$10^{-1}$ & $1^{\prime \mathrm{a}}$ & $101-200$ & $c_{1}=1$ \\
$10^{-2}$ & $1^{\prime \prime \mathrm{b}}$ & $40-100$ & $c_{1}=2$ \\
$10^{-3}$ & & $<50$ & $c_{1}=3$ \\
\hline
\end{tabular}

a One minute of arc.

b One second of arc.

\section{$5 \quad$ Lists}

We have redefined the itemize environment (labelitemi) so that you will receive bullets instead of dashes to introduce the individual items. We think that this way the list

- is clearer

- looks better

- is more noticeable

\section{Appendix}

Of course you may use the standard $\mathrm{LT}_{\mathrm{E}} \mathrm{X}$ command for your appendix. But please be aware of the fact that we have modified so that the numbering of equations, figures and tables remains arabic, see examples below.

$$
\begin{aligned}
& a=c+d, \\
& e=f-d .
\end{aligned}
$$


Table 3. Critical $N$ values

\begin{tabular}{llllll}
\hline $\mathrm{M}_{\odot}$ & $\beta_{0}$ & $T_{\text {c6 }}$ & $\gamma$ & $N_{\text {crit }}^{\mathrm{L}}$ & $N_{\text {crit }}^{\mathrm{Te}}$ \\
\hline 30 & 0.82 & 38.4 & 35.7 & 154 & 320 \\
60 & 0.67 & 42.1 & 34.7 & 138 & 340 \\
120 & 0.52 & 45.1 & 34.0 & 124 & 370 \\
\hline
\end{tabular}

\section{References}

1. W. Frank, A. Seeger: Appl. Phys. A 3, 66 (1988)

2. W. Greiner, D.N. Poenaru: 'Cluster Preformation in Closed- and Mid-shell Nuclei'. In: Atomic and Nuclear Clusters, 2nd International Conference at Santorini, Greece, June 28-July 2, 1993, ed. by G.S. Anagnostatos, W. von Oertzen (Springer, Heidelberg 1994) pp. 264-266

3. F. Holzwarth, J. Lenz et al.: 1readme. Further Details on Layout and $E_{E} T_{E} X$ code. (Springer, Berlin Heidelberg 1999)

4. B. Jirgensons: Optical Activity for Proteins and Other Macro-Molecules, 2nd edn. (Springer, New York 1984)

5. D.M. MacKay: 'Visual Stability and Voluntary Eye Movements'. In: Handbook of Sensory Physiology VII/3. ed. by R. Jung (Springer, Berlin, Heidelberg 1973) pp. 307-331

6. M. Müller, F.J. Becker: On Generalized Hamiltonian Dynamics (Cambridge University Press, Cambridge 1930)

7. S. Nakamura, M. Senoh, N. Iwasa, S. Nagahama: Jpn. J. Appl. Phys. 34, L797 (1995) W. Frank, A. Seeger: Appl. Phys. A 3, 66 (1988)

8. D.W. Ross: Lysosomes and Storage Diseases. MA Thesis, Columbia University, New York (1977) 


\title{
TRAFFIC PHENOMENA IN BIOLOGY:
}

\section{TRAFFIC PHENOMENA IN BIOLOGY: from molecular motors to organisms}

\author{
Debashish Chowdhury*1 \\ ${ }^{1}$ Department of Physics, Indian Institute of Technology, Kanpur 208016, India.
}

Traffic-like collective movements are observed at almost all levels of biological systems. Molecular motor proteins like, for example, kinesin and dynein, which are the vehicles of almost all intra-cellular transport in eukayotic cells, sometimes encounter traffic jam that manifests as a disease of the organism. Similarly, traffic jam of collagenase MMP-1, which moves on the collagen fibrils of the extracellular matrix of vertebrates, has also been observed in recent experiments. Traffic-like movements of social insects like ants and termites on trails are, perhaps, more familiar in our everyday life. Experimental, theoretical and computational investigations in the last few years have led to a deeper understanding of the generic or common physical principles involved in these phenomena. In particular, some of the methods of non-equilibrium statistical mechanics, pioneered almost a hundred years ago by Einstein, Langevin and others, turned out to be powerful theoretical tools for quantitaive analysis of models of these traffic-like collective phenomena as these systems are intrinsically far from equilibrium. In this review we critically examine the current status of our understanding, expose the limitations of the existing methods, mention open challenging questions and speculate on the possible future directions of research in this interdisciplinary area where physics meets not only chemistry and biology but also (nano-)technology.

* E-mail: debch@iitk.ac.in 

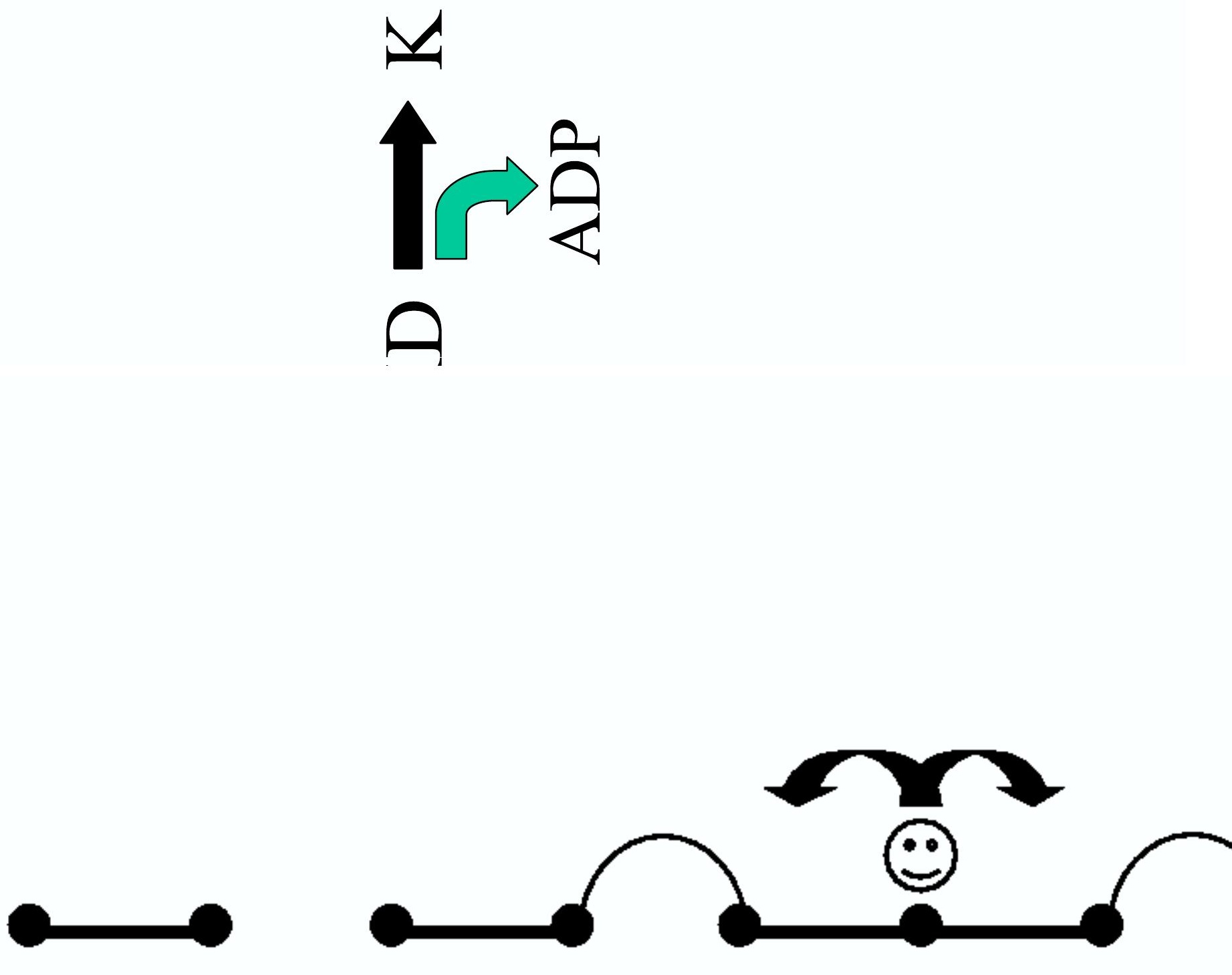